\title{
Publisher Correction: Western US volcanism due to intruding oceanic mantle driven by ancient Farallon slabs
}

Quan Zhou, Lijun Liu and Jiashun Hu

Correction to: Nature Geoscience https://doi.org/10.1038/s41561-017-0035-y, published online 18 December 2017.

In the version of this Article originally published, data points representing mafic eruptions were missing from Fig. $4 \mathrm{~b}$, the corrected version is shown below. Furthermore, the authors omitted to include the following acknowledgements to the provider of the computational resources: "This research is part of the Blue Waters sustained-petascale computing project, which is supported by the National Science Foundation (awards OCI-0725070 and ACI-1238993) and the state of Illinois. Blue Waters is a joint effort of the University of Illinois at Urbana-Champaign and its National Center for Supercomputing Applications. This work is also part of the 'PRAC Title 4-D Geodynamic Modeling With Data Assimilation: Origin Of Intra-Plate Volcanism In The Pacific Northwest' PRAC allocation support by the National Science Foundation (award number ACI 1516586). This work also used the Extreme Science and Engineering Discovery Environment (XSEDE), which is supported by National Science Foundation grant number ACI-1548562." Figure 4 and the Acknowledgements section have been updated in the online version of the Article.
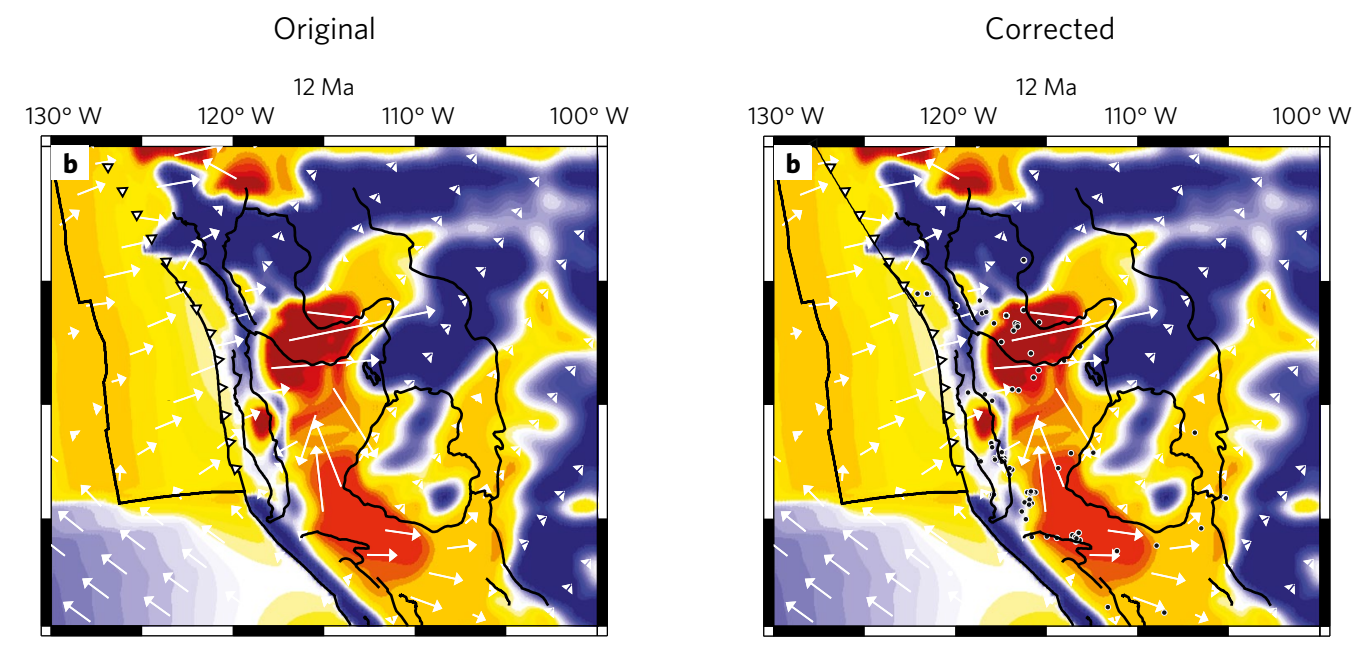

Fig. 4b | Original and corrected. The corrected version includes the mafic data points.

Published online: 19 February 2018

https://doi.org/10.1038/s41561-018-0062-3

\section{Publisher Correction: Puzzling features of western Mediterranean tectonics explained by slab dragging}

Wim Spakman (D, Maria V. Chertova, Arie. P. van den Berg and Douwe J. J. van Hinsbergen

Correction to: Nature Geoscience https://doi.org/10.1038/s41561-018-0066-z, published online 19 February 2018.

In the version of this Article originally published, the author list and journal name were incorrect in ref. 23, the reference should have read: 'Neres, M. et al. Lithospheric deformation in the Africa-Iberia plate boundary: improved neotectonic modeling testing a basal-driven Alboran plate. J. Geophys. Res. Solid Earth 121, 6566-6596 (2016).' This has been corrected in the online versions. 